\title{
Lung-protective Ventilation Combined With Pressure-controlled Ventilation Volume-guaranteed in Children Undergoing One-lung Ventilation: A Randomised Controlled Trial
}

Change Zhu

Shanghai Children's Hospital, Shanghai Jiao Tong University

\section{Saiji Zhang}

Shanghai Children's Hospital, Shanghai Jiao Tong University

Shenghua Yu

Shanghai Children's Hospital, Shanghai Jiao Tong University

\section{Yuting Zhang}

Shanghai Children's Hospital, Shanghai Jiao Tong University

Rong Wei ( $D$ Rongwei2014@163.com )

Shanghai Children's Hospital, Shanghai Jiao Tong University

\section{Research Article}

Keywords: lung-protective ventilation, pressure-controlled ventilation volume-guaranteed, one-lung ventilation, children

Posted Date: September 27th, 2021

DOI: https://doi.org/10.21203/rs.3.rs-902704/v1

License: (c) (i) This work is licensed under a Creative Commons Attribution 4.0 International License.

Read Full License 


\section{Abstract}

The purpose of study was to evaluate the effect of lung-protective ventilation (LPV) combined with PCVVG in children undergoing OLV. Patients were randomly assigned to the LPV combined with PCV-VG group (PCV-VG) or LPV group (volume-controlled ventilation). Both groups received tidal-volume ventilation of $8 \mathrm{ml} \mathrm{kg}^{-1}$ body weight during two-lung ventilation, $6 \mathrm{ml} \mathrm{kg}^{-1}$ during OLV, with sustained 5 $\mathrm{CmH}_{2} \mathrm{O}$ positive end-expiratory pressure. The PCV-VG group exhibited lower PIP than the LPV group at T1 (16.8 \pm 2.3 vs. $18.7 \pm 2.7, P=0.001)$, T2 ( $20.2 \pm 2.7$ vs. $22.4 \pm 3.3, P=0.001)$, and T3 $(23.8 \pm 3.2$ vs. $26.36 \pm 3.7$, $P=0.01)$. Dynamic compliance was higher in the PCV-VG group at $\mathrm{T} 1, \mathrm{~T} 2$, and $\mathrm{T} 3(P=0.01)$. After anaesthesia induction, lung aeration deteriorated, but with no immediate postoperative difference in both groups. Postoperative lung aeration improved and returned to normal from $2.5 \mathrm{~h}$ postextubation in both groups. No differences were observed in postoperative pulmonary complications, intra-operative desaturation, hospital stay. In paediatric patients, who underwent thoracoscopic surgery, PCV-VG combined with LPV was superior to LPV in its ability to provide ventilation with lower PIP and higher dynamic compliance. However, the long-term benefits of different ventilation strategies should be further investigated.

\section{Key Points:}

1. . Evidence-based recommendations for ventilation strategies during OLV in children are lacking.

2. . Studies involving adults have demonstrated that PCV-VG potentially reduces airway pressure and improves lung compliance.

3. . The authors evaluated the effect of lung-protective ventilation combined with PCV-VG in children requiring OLV for thoracoscopic surgery.

4. . PCV-VG combined with LPV was superior to LPV in its ability to provide ventilation with lower PIP and higher dynamic compliance; however, no differences in lung aeration, postoperative pulmonary complications, and intra-operative desaturation was observed.

5. . This approach may facilitate goal-directed trials in peri-operative lung protection for children requiring OLV for thoracoscopic surgery.

\section{Introduction}

One-lung ventilation (OLV) has been widely used in children; however, it is associated with increased postoperative pulmonary complications, ${ }^{1}$ which is recognized as a risk factor for acute lung injury (ALI). ${ }^{2}$ $\mathrm{ALI}$ and acute respiratory distress syndrome are the leading causes of death after thoracic surgery, and they significantly reduce 1 -year survival. ${ }^{3}$ Paediatric patients have smaller functional residual capacities and larger closing volumes, rendering them more susceptible to lung injury during surgery. ${ }^{4}$ Owing to high airway pressures during OLV for pulmonary resection, children are vulnerable to ventilatorinduced barotrauma are of great concern. ${ }^{5}$ PCV-VG is a novel type of ventilation. The decreasing airflow of PCV- 
VG allows airway pressure to achieve its maximum value at the beginning of inhalation and sustains the entire inhalation phase. Continuous plateau pressure in PCV-VG is more conducive for oxygen diffusion. ${ }^{6}$ To date, several studies involving adults have demonstrated that PCV-VG potentially reduces airway pressure and improves lung compliance compared to $\mathrm{VCV} ;{ }^{6}$ however, to the best of our knowledge, no studies have investigated PCV-VG in children undergoing OLV surgery. This study aimed to compare PCVVG combined with LPV with LPV alone in terms of airway pressure, dynamic compliance, lung aeration, postoperative pulmonary complications, intra-operative desaturation, hospital stay, and haemodynamics in children during thoracoscopic surgery.

\section{Methods}

The study was conducted in accordance with the principles of the Declaration of Helsinki ${ }^{7}$ after receiving approval from the Ethics Committee of Shanghai Children's Hospital, Shanghai, China on 24 July 2019 (approval number: 2019R044-F01). The trial was registered in the Chinese Clinical Trial Registry at www.chictr.org.cn (trial number: ChiCTR2000035189, 02/08/2020). Confirms that informed consent was obtained from the parents or legal guardians of the children. This single-centre, prospective, randomised controlled trial was conducted at a tertiary teaching children's hospital affiliated with Shanghai Jiao Tong University, China, from 7 August 2020. The enrolment and allocation of patients are summarised in a CONSORT flow diagram (Fig. 1).

Healthy paediatric patients aged 0-5 years with American Society of Anesthesiologists physical status 1 or 2 who received one lung ventilation during general anaesthesia while undergoing thoracoscopic surgery were included. The exclusion criteria severe heart disease, other lung disease, upper respiratory tract infections, difficult airway or tracheotomy.

Computer-generated, sealed-envelope randomisation was performed to assign patients to one of the following two parallel arms in a 1:1 ratio, receiving different mechanical ventilation protocols: LPV combined with PCV-VG (PCV-VG group) or LPV group (control, volume-controlled ventilation). One investigator (SZ) opened the envelopes and performed different mechanical ventilation protocols. The investigator did not participate in any other aspects of the trial. The patients as well as the Data Safety and Monitoring Board were also blinded to the random allocation.

All patients received a standardised general anaesthetic protocol, which included pre-oxygenation (without continuous positive airway pressure) and intravenous fentanyl $\left(2 \mathrm{mg} \mathrm{kg}^{-1}\right)$, propofol $\left(3 \mathrm{mg} \mathrm{kg}^{-}\right.$ $\left.{ }^{1}\right)$, and rocuronium $\left(0.6 \mathrm{mg} \mathrm{kg}^{-1}\right)$. 5-Fr bronchial blocker (BB) was placed outside the endotracheal tube and placed into the target bronchi using fibre-optic bronchoscope. The pressure of the tracheal intubation cuff was maintained at $20-30 \mathrm{cmH}_{2} \mathrm{O} .{ }^{8}$ All patients received ventilation using the same type of mechanical ventilator. Finally, the lungs were re-expanded manually with sustained inflation with $20-30$ $\mathrm{cmH}_{2} \mathrm{O}$ of positive airway pressure for $10-15 \mathrm{~s}$ under direct observation. ${ }^{9,10}$ 
Anaesthesia was maintained using propofol $5-8 \mathrm{mg} \mathrm{kg}^{-1} \mathrm{~h}^{-1}$ to maintain the BIS (Philips Healthcare, Andover, MA) at 40-60 and remifentanil $0.1-0.4 \mathrm{ug} \mathrm{kg}^{-1} \mathrm{~min}^{-1}$ to maintain haemodynamic stability, and rocuronium $0.3-0.6 \mathrm{mg} \mathrm{kg}^{-1} \mathrm{~h}^{-1}$ was maintained. Crystalloid solutions $\left(6-8 \mathrm{ml} \mathrm{kg}^{-1} \mathrm{~h}^{-1}\right)$ were used as maintenance fluids intra-operatively.

\section{Ventilation settings in both groups were as follows:}

- - Tidal volume of $8 \mathrm{ml} \mathrm{kg}^{-1}$ during two-lung ventilation (TLV) and $6 \mathrm{ml} \mathrm{kg}^{-1}$ during OLV

- Inspiratory/expiratory ratio of 1:2

- - Inspired oxygen concentration of 0.5 with air during TLV and 1 during OLV

- $2.01 \mathrm{Imin}^{-1}$ of inspiratory fresh gas flow

- Positive end-expiratory pressure of $5 \mathrm{cmH}_{2} \mathrm{O}$

To ensure the end-expiratory concentration at 4.7-5.3 $\mathrm{kPa}$, respiratory rate was adjusted at 18-25 breaths/min during TLV and 25-30 breaths min $^{-1}$ during OLV to maintain an $\mathrm{ETCO}_{2}$ of no more than 7.9 $\mathrm{kPa}$.

\section{Measurements:}

Data collections were performed during the following time points:

- Before induction of anaesthesia (T0)

- 10 min after induction of anaesthesia during TLV (T1)

- 5 min after OLV initiation (T2)

- Postpneumoperitoneum (5 min after complete $\mathrm{CO}_{2}$ insufflations) (T3)

- After surgery (T4)

- $2.5 \mathrm{~h}$ after surgery (T5).

\section{The following data were collected or calculated:}

- peak inspiratory pressure (PIP), dynamic compliance

- $\mathrm{PaO}_{2}$ and $\mathrm{PaCO}_{2}$

- Postoperative pulmonary complications

- - Lung ultrasonography (LUS) in the dependent lung

LUS was performed at the following three specific intervals: immediately before induction of anaesthesia (T0), immediately after the surgical procedure (T4), $2.5 \mathrm{~h}$ after surgery (T5).

The four levels of aeration in LUS examination were classified as follows: $N=0, B 1=1, B 2=2$, and $C=$ 3. ${ }^{10,11}$ 


\section{Statistical analysis}

Data are expressed as $n(\%)$, mean $\pm S D$, or median (IQR) depending on the distribution of the data. Comparison of continuous variables between the study groups was performed using Student's t-test for normally distributed data or the Mann-Whitney U-test for non-normally distributed data. Within-group comparisons between the different time points were performed using a paired t-test for normally distributed data. Comparison of the different variables over the study time points was performed using repeated measures analysis of variance. Statistical significance was set at $p<0.05$.

A power analysis suggested that a minimum sample size of 26 patients for each group would be required to achieve a significance level of $5 \%$ with a power of $80 \%$. The power was calculated from our preliminary data using an independent t-test, and the difference in mean peak inspiratory pressure between both modes of ventilation was $3 \mathrm{cmH}_{2} \mathrm{O}$, with a standard deviation of $3.8 \mathrm{cmH}_{2} \mathrm{O}$ during OLV. The dropout rate was $20 \%$, and 63 patients were sufficient. All statistical calculations were performed using the computer program SPSS version 25 (Statistical Package for the Social Science; IBM, Armonk, NY).

\section{Results}

Patient enrolment commenced on 7 August 2020. In total, 63 patients were randomly assigned to the LPV $(n=31)$ and PCV-VG $(n=32)$ groups (Fig. 1). The baseline characteristics did not differ between the groups (Table 1).

Table 1

Baseline characteristics

\begin{tabular}{|llll|}
\hline Parameters & $\begin{array}{l}\text { LPV group } \\
(\boldsymbol{n}=\mathbf{3 1})\end{array}$ & $\begin{array}{l}\text { PCV-VG group } \\
(\boldsymbol{n}=\mathbf{3 2})\end{array}$ & P-value \\
\hline Age (month) & $6.4[5-40.75]$ & $6.8[5.2-39.3]$ & 0.755 \\
\hline Weight (kg) & $8.3[7.5-13.25]$ & $9[7.55-12.3]$ & 0.705 \\
\hline Sex (male) & $25 / 6$ & $24 / 8$ & 0.59 \\
\hline Type of operation & & & \\
\hline Segmentectomy/ & 27 & 25 & 0.35 \\
wedge resection & & & 0.72 \\
\hline Single lobectomy & 3 & 4 & 0.3 \\
\hline Bilobectomy & 1 & 3 & \\
\hline
\end{tabular}

All data are presented as mean \pm SD or median [IQR], unless otherwise specified.

LPV, lung-protective ventilation; PCV-VG, pressure-controlled ventilation volume-guarantee 


\section{Primary outcome}

PIP at T2 and T3 were lower in the PCV-VG group than in the LPV group (T2, 20.2 $\pm 2.7 v s 22.4 \pm 3.3$; $P=0.001)(T 3,23.8 \pm 3.2 v s 26.36 \pm 3.7 ; P=0.01)$ (Fig. 2A). Dynamic compliance at $T 2$ and $T 3$ was higher in the PCV-VG group than in the LPV group $(T 2,9.1 \pm 3.7$ vs $6.8 \pm 3.05 ; P=0.01)(T 3,7.1 \pm 3.3$ vs $4.8 \pm 2.3$; $\mathrm{P}=0.01$ ) (Fig. 2B).

In addition,PIP was higher at T2 and T3 compared with T1( $P=0.001)$ (Fig. 2A). Dynamic compliance was lower at $\mathrm{T} 2$ and $\mathrm{T} 3$ compared with $\mathrm{T} 1(P=0.01)$ (Fig. 2B).

\section{Secondary outcomes}

\section{LUS assessment}

There was no difference in LUS in the dependent lung before T0. After T0, lung aeration deteriorated, but with no difference in both groups immediately after surgery [T4: 4 ( 2 to 6 ) vs. 4 ( 2 to 5 ) $Z=-0.69, P=0.49$ ] (Figure $3 \mathrm{a}$ and Figure $3 \mathrm{c}$ ). Lung aeration improved in both groups after surgery and returned to normal from $2.5 \mathrm{~h}$ after extubation in both groups (Figure $3 \mathrm{~b}$ and Figure $3 \mathrm{~d}$ ). Temporal ultrasound images of the lateral chest wall of the dependent lung are shown in Fig. 3.

\section{Postoperative pulmonary complications}

Five (7.9\%) patients exhibited Postoperative pulmonary complications occurred in five patients, with no differences in the incidence between the two groups [LPV: 3 (9.7\%) vs. PCV-VG: $2(6.3 \%), P=0.97$ ].

\section{Intra-operative desaturation}

Intra-operative desaturation was comparable in LPV group $(4 / 31,12.9 \%)$, compared with $3 / 32(9.4 \%)$ in the PCV-VG group [OR=1.43 (0.29 to 7.0); $P=0.66]$.

There was no difference in the gas exchange values between the groups. $\mathrm{PaO}_{2}$ and $\mathrm{PaCO}_{2}$ increased significantly at T3 compared with that at baseline $(P=0.001)$ (Table 2$)$.

\section{Table 2. Gas exchange}

\begin{tabular}{|llll|}
\hline Variable & Group & $\mathrm{T} 1$ & $\mathrm{~T} 3$ \\
\hline $\mathrm{PaO}_{2}$ & LPV & $214.6 \pm 23.67$ & $249.25 \pm 19.14$ \\
$(\mathrm{mmHg})$ & $\mathrm{PCV}-\mathrm{VG}$ & $218.85 \pm 19.74$ & $258.82 \pm 25.19$ \\
$\mathrm{PaCO}_{2}$ & LPV & $36.18 \pm 6.4$ & $54.92 \pm 8.44$ \\
\cline { 2 - 4 }$(\mathrm{mmHg})$ & PCV-VG & $35.70 \pm 5.22$ & $51.74 \pm 10.15$ \\
\hline
\end{tabular}

All data are presented as mean \pm SD. 
$\mathrm{PaCO}_{2}$, arterial pressure of carbon dioxide; $\mathrm{PaO}_{2}$, arterial pressure of oxygen; LPV, lung-protective ventilation; PCV-VG, pressure-controlled ventilation volume-guaranteed ventilation; T1, 10 min after induction of anaesthesia without pneumoperitoneum; $\mathrm{T} 3,5$ min after complete $\mathrm{CO}_{2}$ insufflation

\section{Hospital stays}

The length of hospital stay did not differ between the PCV-VG $(6.5 \pm 2.1)$ and VCV $(6.1 \pm 1.9)$ groups [0.4 (95\% $\mathrm{Cl}-0.59$ to 1.39$), P=0.43]$.

\section{Haemodynamic variables}

There was no difference in the haemodynamic variables between the groups. MAP was higher at T3 in both groups compared with that at T1 and T2 $(P=0.001)$. CVP was higher at T2 and T3 than that at T1 $(P=0.001)$. The heart rate was stable throughout the operation (Fig. 4).

\section{Discussion}

This randomised controlled trial revealed that PCV-VG reduced airway pressure and increased dynamic compliance than VCV in children undergoing thoracoscopic surgery. After induction of anaesthesia, lung aeration deteriorated; however, there was no difference in either group immediately after surgery. Lung aeration improved in both groups after surgery and returned to normal from $2.5 \mathrm{~h}$ after extubation in both groups. There was no difference in postoperative pulmonary complications, oxygenation, hospital stay, or haemodynamics.

Similar results have been obtained in laparoscopic ${ }^{6,13}$ and lumbar spine ${ }^{14}$ surgeries. High airway pressures achieved during OLV have reportedly been associated with postpneumonectomy pulmonary oedema ${ }^{4,15}$ and acute lung injury after pneumonectomies. ${ }^{16}$ Therefore, patients undergoing OLV may benefit more from lower PIP. To the best of our knowledge, evidence-based recommendations for ventilation strategies during OLV in children are lacking and this is the first randomised controlled trial to evaluate the effect of LPV combined with PCV-VG during OLV in a paediatric population.

Patients who received PCV-VG exhibited lower PIP and higher dynamic compliance, indicating a possible association with the decreasing airflow of PCV-VG, which allows airway pressure to achieve its maximum at the beginning of inhalation and continue the entire inspiratory phase. ${ }^{6}$ Continuous measurement of lung compliance and volumetric pressure automatically adjusts the air supply flow rate and air pressure. ${ }^{6}$ Therefore, PCV-VG mode potentially reduces airway pressure to the greatest extent possible while ensuring ideal volume and improving lung compliance. ${ }^{11}$

In the current study, we found no difference in PPCs between the two groups. This may be related to limited fluid input, plateau pressures remaining below $30 \mathrm{cmH}_{2} \mathrm{O}$ at all times, ${ }^{17}$ and lung-protective ventilation. ${ }^{15}$ Most important, the sample was too small. 
We also found that lung aeration was comparable in both groups after surgery and was fully restored 2.5 $\mathrm{h}$ after surgery, as evaluated using LUS. This result is consistent with that of our previous study. ${ }^{12}$ However, the influence of the ventilation mode can easily overlap because patient factors or other factors affect patient prognosis to a greater extent. ${ }^{18}$ For instance, the lungs were manually re-expanded at the time of thoracic cavity closure. Moreover, patient may exhibit compensatory adaptations, which need to be considered. ${ }^{18}$ To be more specific, patient with healthy lungs may compensate and overcome perioperative lung problems, such as lung oedema or atelectasis, but this is unlikely in patients with ALI or acute respiratory distress syndrome,

$\mathrm{PaO}_{2}$ increased significantly in both groups at $\mathrm{T} 3$ compared to that at baseline, and no superiority in oxygenation was observed regardless of the mode of ventilation, a finding that corroborates with a previous study. ${ }^{19}$ This finding may be explained by the similarity in the mean values. ${ }^{13}$

However, this study also had certain limitations. First, blinding was not conducted in investigators who were aware of the mode of ventilation. Second, we did not enrol patients with obesity or lung injuries. In summary, PCV-VG mode reduced the airway pressure and increased dynamic compliance in patients who underwent thoracoscopic surgery requiring OLV. However, its benefificial effects on lung aeration, postoperative pulmonary complications,

intra-operative desaturation and hospital stay remain unclear.

\section{Declarations}

\section{Acknowledgments relating to this article}

Assistance with this article: We thank our colleagues within the Department of Anesthesiology, Shanghai Children's Hospital, Shanghai, China (Yuezhen Fu, MD, PHD and Yiru Tong, MD, PHD) for technical assistance.

\section{Author contributions}

Study design/planning: Rong Wei and Change Zhu. Study conduct: Saiji Zhang and Change Zhu. Data collection: Shenghua Yu and Yuting Zhang. Writing the paper: Change Zhu and Rong Wei. Confrming the fnal paper: all authors.

\section{Competing interests}

Te authors declare no competing interests.

Financial support and sponsorship: Funding from Special Clinical Research Project of Shanghai Municipal Health Commission (20204Y0470) 


\section{References}

1. Licker, M. J. et al. Operative mortality and respiratory complications after lung resection for cancer: impact of chronic obstructive pulmonary disease and time trends. Ann Thorac Surg, 81, 1830-1837 (2006).

2. Lohser, J. Evidence-based management of one-lung ventilation. Anesthesiol Clin, 26, 241-272 (2008).

3. Fernández-Pérez, E. R. et al. Intraoperative ventilator settings and acute lung injury after elective surgery: a nested case control study. Thorax, 64, 121-127 (2009).

4. Lee, J. H. et al. Lung protective ventilation during pulmonary resection in children: a prospective, single-centre, randomised controlled trial. Br J Anaesth, 122, 692-701 (2019).

5. Park, M. et al. Driving Pressure during Thoracic Surgery: A Randomized Clinical Trial., 130, 385-393 (2019).

6. Assad, O. M., El Sayed, A. A. \& Khalil, M. A. Comparison of volume-controlled ventilation and pressure-controlled ventilation volume guaranteed during laparoscopic surgery in Trendelenburg position. J Clin Anesth, 34, 55-61 (2016).

7. Preckel, B. et al. Ten years of the Helsinki Declaration on patient safety in anaesthesiology: an expert opinion on peri-operative safety aspects. Eur J Anaesthesiol, 37, 521-610 (2020).

8. Yan, J., Rufang, Z., Rong, W. \& Wangping, Z. Extraluminal Placement of the Bronchial Blocker in Infants Undergoing Thoracoscopic Surgery: A Randomized Controlled Study. J Cardiothorac Vasc Anesth, 34, 2435-2439 (2020).

9. Acosta, C. M. et al. Lung recruitment prevents collapse during laparoscopy in children: A randomised controlled trial. Eur J Anaesthesiol, 35, 573-580 (2018).

10. Templeton, T. W., Piccioni, F. \& Chatterjee, D. An Update on One-Lung Ventilation in Children. Anesth Analg, 132, 1389-1399 (2021).

11. Acosta, C. M. et al. Accuracy of transthoracic lung ultrasound for diagnosing anesthesia-induced atelectasis in children. Anesthesiology, 120, 1370-1379 (2014).

12. Zhu, C., Zhang, S., Dong, J. \& Wei, R. Effects of positive end-expiratory pressure/recruitment manoeuvres compared with zero end-expiratory pressure on atelectasis in children as tracked by ultrasound: A randomised clinical trial.Eur J Anaesthesiol. Jan28. (2021).

13. Lee, J. M. et al. Comparison of volume-controlled, pressure-controlled, and pressure-controlled volume-guaranteed ventilation during robot-assisted laparoscopic gynecologic surgery in the Trendelenburg position. Int J Med Sci, 17, 2728-2734 (2020).

14. Lee, J. M. et al. Comparison of volume-controlled ventilation mode and pressure-controlled ventilation with volume-guaranteed mode in the prone position during lumbar spine surgery. BMC Anesthesiol, 19, 133 (2019).

15. Lohser, J. \& Slinger, P. Lung Injury After One-Lung Ventilation: A Review of the Pathophysiologic Mechanisms Affecting the Ventilated and the Collapsed Lung. Anesth Analg, 121, 302-318 (2015). 
16. Yang, M. et al. Does a protective ventilation strategy reduce the risk of pulmonary complications after lung cancer surgery?: a randomized controlled trial. Chest, 139, 530-537 (2011).

17. Amar, D. et al. Protective Lung Ventilation and Morbidity After Pulmonary Resection: A Propensity Score-Matched Analysis. Anesth Analg, 125, 190-199 (2017).

18. Kim, Y. S. et al. Lung ultrasound score-based perioperative assessment of pressure-controlled ventilation-volume guaranteed or volume-controlled ventilation in geriatrics: a prospective randomized controlled trial. Clin Interv Aging, 14, 1319-1329 (2019).

19. Aldenkortt, M. et al. Ventilation strategies in obese patients undergoing surgery: a quantitative systematic review and meta-analysis. Br J Anaesth, 109, 493-502 (2012).

\section{Figures}




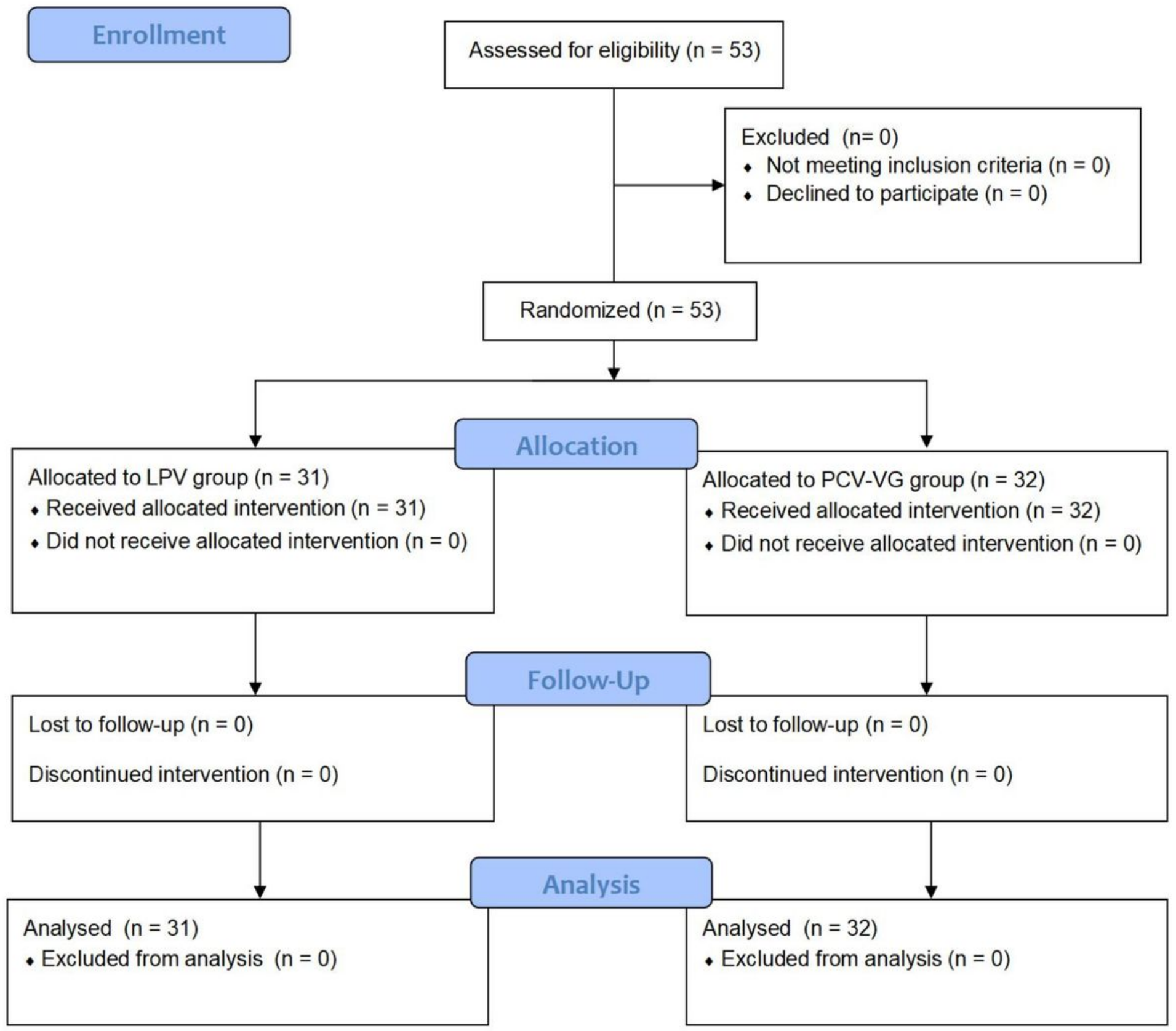

Figure 1

CONSORT flow diagram for patients included in the study 

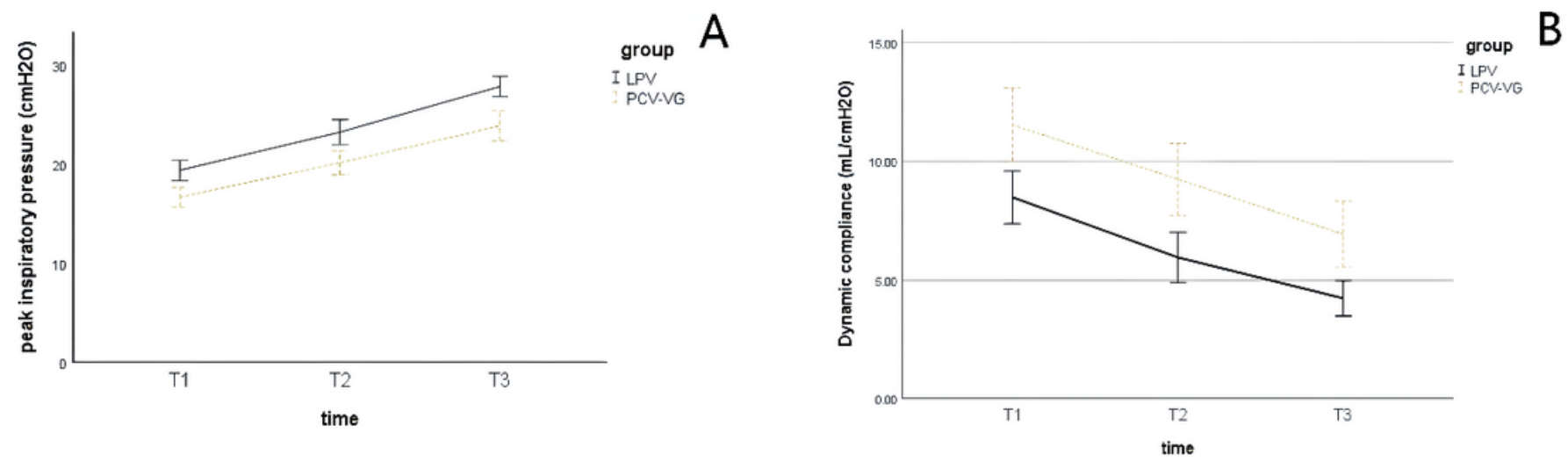

Figure 2

Peak inspiratory pressure and dynamic compliance in the two groups at different stages of the study. Figure 2A. peak inspiratory pressure Figure 2B. dynamic compliance $\mathrm{T} 1,10 \mathrm{~min}$ after induction of anaesthesia in the supine position without pneumoperitoneum; T2, 5 min after OLV commencement; T3, 5 min after complete $\mathrm{CO} 2$ insufflations; LPV, lung-protective ventilation; PCV-VG, pressure-controlled ventilation volume-guaranteed ventilation 


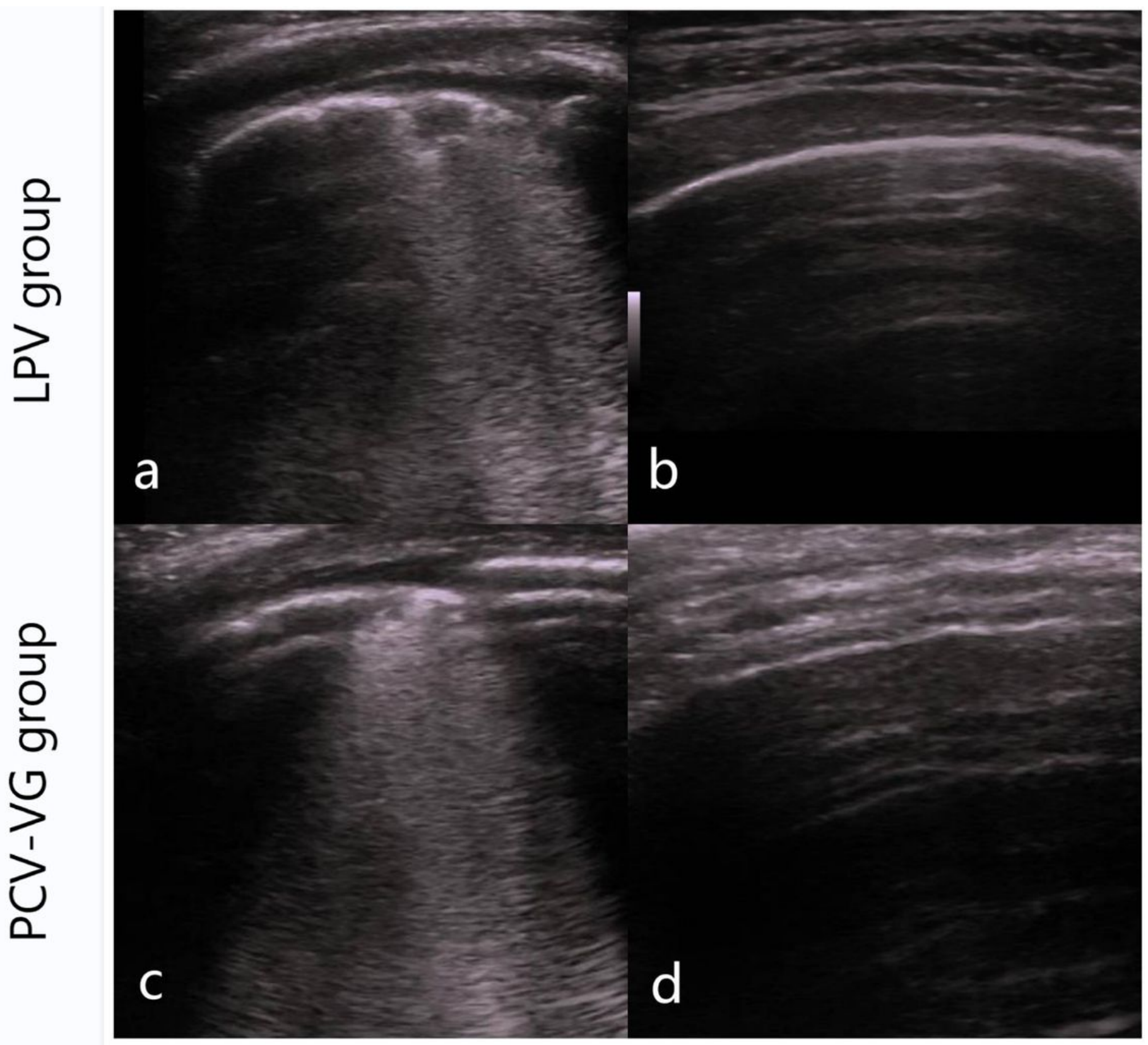

\section{Figure 3}

Lung ultrasound findings of dependent lung in the PCV-VG and LPV groups. The images were obtained from the posterior region of the dependent lung. Figure 3a. Lung aeration after surgery in LPV group. Figure 3c. Lung aeration after surgery in PCV-VG group. Figure 3b. Lung aeration $2.5 \mathrm{~h}$ after surgery in LPV group. Figure 3d. Lung aeration 2.5h after surgery in PCV-VG group. LPV, lung-protective ventilation; PCV-VG, pressure-controlled ventilation volume-guaranteed ventilation 


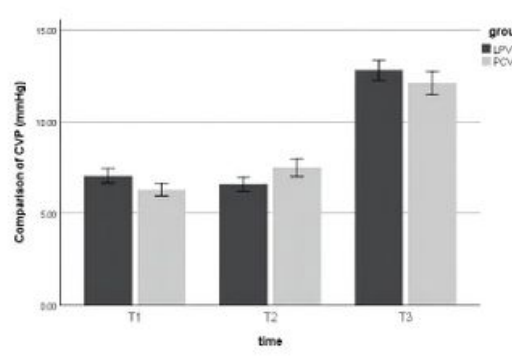

A

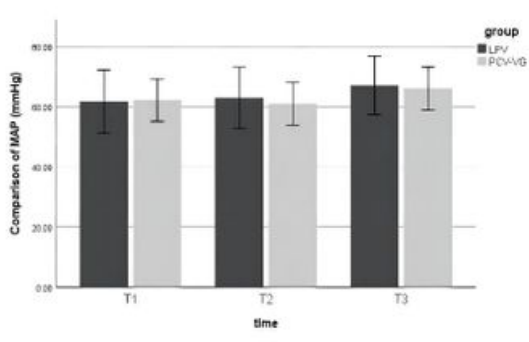

B

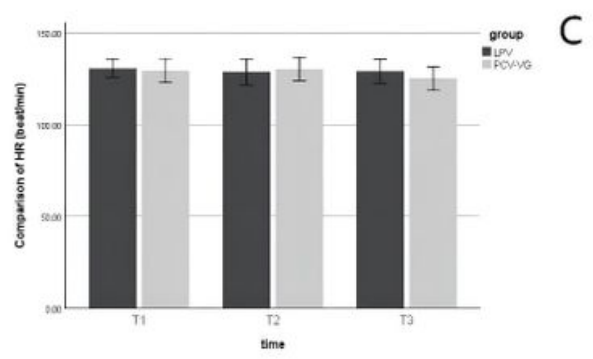

Figure 4

Comparison of haemodynamic variables at different timepoints. Figure 4A.CVP, central venous pressure; Figure 4B.MAP, mean arterial pressure; Figure 4C.HR, heart rate LPV, lung-protective ventilation; PCV-VG, pressure-controlled ventilation volume-guaranteed; $\mathrm{T} 1,10 \mathrm{~min}$ after induction of anaesthesia without pneumoperitoneum; T2, 5 min after OLV commencement; T3, 5 min after complete $\mathrm{CO} 2$ insufflation 